\title{
Bone mineral and hormone status in paraplegics
}

\author{
V Finsen $\mathrm{MD} \mathrm{PhD},{ }^{1} \mathrm{~B}$ Indredavik $\mathrm{MD},{ }^{2} \mathrm{~K} \mathrm{~J}$ Fougner $\mathrm{MD}^{3}$ \\ ${ }^{1}$ Department of Orthopaedic Surgery, ${ }^{2}$ Department of Internal Medicine, ${ }^{3}$ Department of \\ Clinical Chemistry, Trondheim University Hospital, 7006 Trondheim, Norway.
}

Nineteen men who had suffered permanent paraplegia a median of 4 years previously were studied. Eight also had varying degrees of neurological deficit of the upper extremities. Bone mineral, biochemical and hormonal values were compared to those in an age-matched control group in order to detect evidence of systemic osteopenia. There were very considerable individual variations in bone mineral density (BMD) deficits among patients compared to controls, probably partly due to methodological problems. Significant BMD deficits were found in the metaphysis $(45 \%)$ and diaphysis $(26 \%)$ of the tibia, whi!e the deficit of the distal forearm was barely significant for the group as a whole. There was a negative correlation between time since injury and degree of BMD deficit in the lower extremity. Those with neurological affection of the upper extremities had a greater BMD deficit of their arms than those with neurologically intact arms. It was concluded that osteopenia in paraplegics is largely confined to the paralysed extremities, and thus not systemic.

Serum alanine aminotransferase, phosphate, follicle stimulating hormone, and free androgen index (testosterone/sex hormone binding globulin) were mainly within normal limits, but significantly higher in paraplegics than in controls. Osteopenia in these patients is thus not due to gonadal dysfunction.

Key words: paraplegia; spinal cord injury; bone mineral density; osteopenia.

\section{Introduction}

Healthy individuals who are confined to bed for long periods lose bone mineral from their skeletons. When ambulation is resumed a full restitution is seen of both diaphyseal bone, which is almost exclusively cortical, and metaphyseal bone, which has a varying amount of trabecular bone. ${ }^{1,2}$ This indicates that this transient osteopenia is probably due to disuse. Patients who suffer spinal cord injuries, however, undergo a period of increased bone turnover where loss of bone mineral greatly exceeds that found with simple bedrest. ${ }^{3,4}$ Griffiths et $a l^{5,6}$ reported that while diaphyseal bone mineral content in the upper limbs of paraplegic patients returns to normal after rehabilitation, the metaphyseal bone mineral content does not. This finding seems to raise the possibility of a systemic factor promoting osteopenia of metaphyseal bone in paraplegic patients.

The purpose of the present case control study was to evaluate bone mineral content in both the upper and lower limbs of men with inveterate traumatic spinal cord lesions and to determine whether osteopenia in such patients could be due to gonadal dysfunction, as it has been established that bone mineral mass is also dependent on sex hormone levels in men. ${ }^{7,8}$

\section{Patients and methods}

Nineteen male patients with spinal cord injuries were studied. Their median age at the time of injury was 20 (range: 15-64) years. They had sustained injuries of the spinal cord a median of 4 years (range: 7 months - 33 years) before study. Levels of fracture and lowest functioning spinal segment are shown in Figure 1. Ten patients had been injured in road traffic accidents, 5 at work, 2 while skiing and 2 by gunshots.

Nine patients were on no regular medication. One patient received buprenorphine 
and one trimetoprim. The remaining 8 patients were on hippuric acid and vitamin C. Two of these also took baclofen.

Eleven patients rarely or never assumed an erect posture. Four were upright between one and 2 hours per week and 4 between 4 and 7 hours.

A male control of the same age was selected for every index patient among nurses, medical students and blood donors. A control for the oldest index patient $(60$ years of age) was selected among our inpatients. A few of the younger controls were a year older or younger than the index patient, while the oldest control was 2 years older than his index patient. None of the controls were on regular medication.

Median height both for index patients before injury and controls at the time of study was $180 \mathrm{~cm}$. The individual index patients were a median of $2 \mathrm{~cm}$ taller $(95 \%$ confidence range: $-7 \mathrm{~cm}$ to $+7 \mathrm{~cm} ; p=$ 0.40 ) than their controls.

Median present weight was $70.5 \mathrm{~kg}$ for index patients and $75 \mathrm{~kg}$ for controls. Individual index patients were a median of $4.5 \mathrm{~kg}$ lighter $(-17 \mathrm{~kg}-+10 \mathrm{~kg} ; p=0.21)$ than controls.

Bone mineral density (BMD) of the upper and lower extremities was determined with a Gambro photonabsorption scanner ${ }^{9}$ which had been modified to also admit the lower limb. The americium 241 beam was collimated to $3 \mathrm{~mm}$ for measurements of the

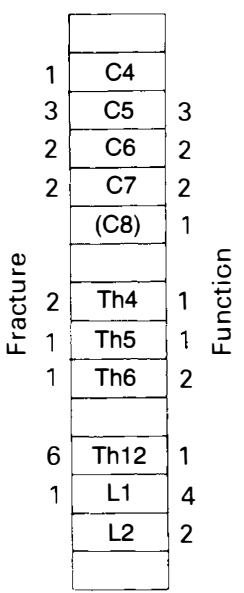

Figure 1 Levels of fracture and lowest functioning spinal segment. arm and $7 \mathrm{~mm}$ for the leg. A constant distance was maintained between source and detector for all measurements at each scan level. Measurements were performed $1 \mathrm{~cm}$ and $10 \mathrm{~cm}$ proximal to the tip of the ulnar styloid and $4 \mathrm{~cm}$ and $10 \mathrm{~cm}$ proximal to the tip of the medial malleolus. The reference points were found by palpation. The non dominant side was measured unless there had been a previous fracture of the extremity. Only the tibia was included in scans of the diaphysis of the leg. At all other scan levels both bones were included. Bone mineral content was divided by the width of the bone to give BMD. Measurements on 2 different days in 11 controls showed the precision of these measurements to be $7 \%$ in the diaphysis and $4 \%$ in the metaphysis of the lower limb and $4 \%$ in the diaphysis and $11 \%$ in the metaphysis of the arm.

The difference in BMD between index patient and control was expressed as a percentage of the BMD of the control and considered negative when BMD was lowest in the index patient. In the 11 controls who had been measured twice the mean values were used.

The biochemical and hormone level determinations which were performed are shown in Table I. All hormone analyses were performed in duplicate with standard radioimmunoassay techniques. Blood for total testosterone determination was drawn before noon, but it was not required that patients should be fasting when blood samples were taken.

Non parametric tests (Wilcoxon's paired test and Kendall's tau) were used for statistical evaluation. One-tailed $p$-values lower than 0.05 were considered significant.

\section{Results}

There was considerable variation in the degree of difference in BMD between index patient and control. This was particularly true for metaphyseal measurements. There was a considerable and significant deficit in BMD in index patients' lower limbs (Table II).

In the arm, however, the diaphyseal deficit was slight and barely significant, while the median metaphyseal deficit was 
Table I Median biochemical and hormone values in index patients and controls. Reference values in brackets. Index-control: values in individual index patients minus that found in their controls

\begin{tabular}{|c|c|c|c|c|}
\hline & Medi & $95 \%$ confid & range & \\
\hline & Index & Control & Index-control & $p=$ \\
\hline Haemoglobin & 15.0 & 14.8 & 0.25 & .264 \\
\hline$(13.5-16.5 \mathrm{~g} / 100 \mathrm{ml})$ & $14.3-15.7$ & $13.5-15.9$ & $-0.9-+1.4$ & \\
\hline Erythrocyte sedimentation & 12 & 5 & +5 & .104 \\
\hline rate $(<13 \mathrm{~mm} /$ hour $)$ & $9-16$ & $3-13$ & $-1-+9$ & \\
\hline Serum creatinine & 67 & 88 & -20 & .0001 \\
\hline$(60-120 \mathrm{umol} / \mathrm{l})$ & $58-73$ & $79-93$ & $-32--8$ & \\
\hline Alanine aminotransferase & 23.5 & 18 & +3 & .007 \\
\hline$(<30 \mathrm{U} / \mathrm{l})$ & $17-39$ & $13-26$ & $2-24$ & \\
\hline Alkaline phosphatase & 183 & 149.5 & 5.5 & .236 \\
\hline$(<270 \mathrm{U} / 1)$ & $116-223$ & $125-182$ & $-14-+57$ & \\
\hline Calcium & 2.35 & 2.42 & -0.07 & .125 \\
\hline$(2.20-2.60 \mathrm{mmol} / \mathrm{l})$ & $2.23-2.52$ & $2.32-2.49$ & $-0.17-+0.08$ & \\
\hline Phosphate & 1.12 & 1.04 & +0.1 & .014 \\
\hline$(0.80-1.50 \mathrm{mmol} / \mathrm{l})$ & $0.96-1.17$ & $0.85-1.09$ & $+0.02-+0.19$ & \\
\hline Albumin & 48 & 50 & -3 & .079 \\
\hline $\begin{array}{l}(35-55 \mathrm{~g} / 1) \\
\text { Parathyroid hormone }\end{array}$ & $\begin{array}{l}45-51 \\
57\end{array}$ & $\begin{array}{c}46-52 \\
54\end{array}$ & $\begin{array}{c}-6-+2 \\
+4\end{array}$ & 480 \\
\hline$(29-85 \mathrm{pmol} / \mathrm{l})$ & $51-60$ & $45-64$ & $-8-+8$ & . 480 \\
\hline Luteinising hormone & 5.8 & 3.9 & +0.8 & .192 \\
\hline (2-12 IU/1, IRP 68/40) & $3.1-7.8$ & $3.1-6.1$ & $-2.1-+3.0$ & \\
\hline Follicle stimulating & 4.4 & 3.4 & +1.3 & .016 \\
\hline $\begin{array}{l}\text { hormone }(2-12 \text { IU/1, IRP } \\
78 / 549)\end{array}$ & $2.3-6.1$ & $2.1-4.0$ & $-0.4-+2.0$ & \\
\hline Testosterone (total) & 21.9 & 22.1 & +2.1 & .367 \\
\hline$(12-40 \mathrm{nmol} / \mathrm{l})$ & $17.6-26.1$ & $15.8-25.1$ & $-4.5-+4.4$ & \\
\hline Sex hormone binding & 22 & 26 & -12 & .009 \\
\hline globulin $(10-50 \mathrm{nmol} / \mathrm{l})$ & $13-31$ & $22-49$ & $-22--4$ & \\
\hline Free androgen index $=$ & 0.85 & 0.62 & +0.365 & .011 \\
\hline testosterone/SHBG & $0.67-1.74$ & $0.47-0.84$ & $+0.095-+1.27$ & \\
\hline
\end{tabular}

Table II Median (95\% confidence range) difference in bone mineral density between index patients and controls

\begin{tabular}{lll}
\hline & $p=$ \\
\hline Tibia distal diaphysis & $-26 \%(-44 \%--19 \%)$ & .0001 \\
Tibia distal metaphysis & $-45 \%(-58 \%--16 \%)$ & .0001 \\
Forearm distal diaphysis & $-5 \%(-10 \%-+3 \%)$ & .0418 \\
Forearm distal metaphysis & $-13 \%(-29 \%-+30 \%)$ & .1611 \\
\hline
\end{tabular}

somewhat more pronounced, but not statistically significant. The 8 patients with partial or complete loss of power in the upper limbs had a median diaphyseal BMD deficit of $8 \%$ ( $95 \%$ confidence range: $-34 \%-+3 \%$ ) and a median metaphyseal deficit of $23 \%$ $(-58 \%-+40 \%)$ in the arm. In contrast, patients with full function of the arms had a median BMD deficit of $3 \%(-14 \%-$ $+26 \%)$ of the diaphysis and $4 \%$ $(-47 \%-+37 \%)$ of the metaphysis. The difference was almost statistically significant for the diaphysis $(p=0.053)$, while the $p$ value was 0.108 for the metaphysis. 
There was no correlation between time of injury and degree of BMD deficit in the upper extremity (tau $>-0.2$ ), but the correlation coefficients for the lower limb were, $-0.70(p<0.001)$ for metaphyseal bone and $-0.55(p<0.01)$ for diaphyseal bone.

Serum creatinine was significantly lower in index patients then in controls, while alanine aminotransferase (ALAT), serum phosphate, follicle stimulating hormone (FSH) and sex hormone binding globulin (SHBG) were lower in controls (Table I). There was no significant differences in the levels of haemoglobin, ESR, alkaline phosphatase (ALP), total calcium, albumin, parathyroid hormone $(\mathrm{PTH})$ and luteinising hormone $(\mathrm{LH})$. The concentrations of total testosterone were practically identical. However, when total testosterone values were divided by SHBG to give an estimate of free testosterone (free androgen index, FAI), this was found to be significantly higher in index patients.

\section{Discussion}

In spite of being matched only with regard to age, patients and controls were of comparable height. Patients were lighter than controls, although not significantly so.

The BMD estimations we have performed are not very precise, but sufficiently so when studying groups of individuals.

The main bone mineral loss was found in the lower limbs, while there was only moderate affection of the upper limbs in the group as a whole. The more pronounced loss seen in the upper limbs of quadriplegic patients would seem to indicate that osteopenia is not generalised, but confined to the neurologically affected extremities. Any initial osteopenia of neurologically intact upper extremities had been almost completely reversed by the time of our measurements.

In the affected limbs, osteopenia was more pronounced in metaphyseal than in diaphyseal bone. This has also been observed in post traumatic osteopenia. ${ }^{10}$ Also the finding of a much more pronounced osteopenia in paralysed lower limbs than paralysed upper limbs has been reported previously. ${ }^{11}$

It was, however, surprising to find that the degree of osteopenia in the affected limbs increased with the time since injury. One might have expected the bones to adjust to the lack of stimulus and reach a lower steady state during the first months or years after the injury. This finding needs corroboration before conclusions can be drawn.

Our finding of a lower serum creatinine among paraplegic patients than in controls is probably due to reduced muscle mass, and confirms a previous report by Mirahmadi et al. ${ }^{12}$ The reason for the significantly raised ALAT is unclear. None of the drugs taken by the patients are known to be deleterious to the liver. The participants in the study were not questioned about alcohol consumption or any sporadic use of analgesics which might affect the liver.

Neither patients nor controls had serum phosphate values outside the reference values of our laboratory, but the median value was significantly higher in patients than in controls. This was not due to low calcium values among patients. Burr and Nuseibeh $^{13}$ found no significant differences in either serum calcium or phosphate values in the paraplegics and controls they studied.

Our findings of no significant difference in LH or total testosterone, but higher levels of $\mathrm{FSH}$ in patients than controls are not in accordance with previous reports. Perkash et $a l^{14}$ found raised $\mathrm{LH}$ and total testosterone in paraplegic patients while FSH was similar to that in controls. Naftchi ${ }^{15}$ reported total testosterone and $\mathrm{FSH}$ to be the same in spinal cord injured patients and controls while LH was lower in quadriplegics than in paraplegics and controls. ClausWalker et al ${ }^{16}$ found lowered total testosterone values for the first 18 months after paralysis, and a tendency toward normalisation with time. Phelps et al $^{17}$ reported total testosterone, free testosterone and serum sex binding protein well within the reference ranges in 50 men with spinal cord injuries. They found somewhat higher total plasma testosterone in quadriplegics than in paraplegics, but there were no significant differences in free testosterone, serum sex binding protein, or percent of free testosterone. It may be of importance that none of these reports were based on case control studies. 
Among our patients serum SHBG was lower than in controls, although within the normal range. For this reason, however, the free androgen index was significantly higher in index patients, and thus the level of free testosterone. ${ }^{18}$ None of the patients had, to our knowledge, received androgenic or ana- bolic steroid therapy. The significance of these findings is unclear, and would need to be confirmed before hypotheses are formulated. They do not, however, indicate that gonadal dysfunction is a factor in the aetiology of osteopenia among paraplegic patients.

\section{References}

1 Donaldson CL, Hulley SB, Vogel JM, Hattner RS, Bayers JH, McMillan DE (1970) Effect of prolonged bed rest on bone mineral. Metabolism 19: 1071-1084.

2 Hulley SB, Vogel JM, Donaldson CL, Bayers JH, Friedman RJ, Rosen SN (1971) The effect of supplemental oral phosphate on the bone mineral changes during prolonged bed rest. J Clin Invest 50: 2506-2518.

3 Claus-Walker J, Campos RJ, Carter RE, Vallbona C, Lipscomb HS (1972) Calcium excretion in quadriplegia. Phys Med Rehabil 53: 14-20.

4 Naftchi NE, Viau AT, Sell GH, Lowman EW (1980) Effect of thyrocalcitonin on 18 F uptake and various biochemical variables in spinal cord injured subjects with periarticular bone formation. In: Mazess RB, ed. Proceedings Fourth International Conference on Bone Measurement. US Department of Health and Human Sciences. NIH publication no. 80-1938: 467-477.

5 Griffith HJ, D’Orsi CJ, Zimmerman RE (1972) Use of 125 I photon scanning in the evaluation of bone density in a group of patients with spinal cord injury. Invest Radiol 7: 107-111.

6 Griffith HJ, Bushueff B, Zimmerman RE (1976) Investigation of the loss of bone mineral in patients with spinal cord injury. Paraplegia 14: 207-212.

7 Foresta C, Ruzza G, Mioni R, Guarneri G, Gribaldo R, Maneghello A et al (1984) Osteoporosis and decline of gonadal function in the elderly male. Hormone Res 19: 18-22.

8 Peacock M, Horsman A. Marshall DH, Aaron JE, Francis RM, Selby PL (1984) Senile and postmenopausal osteoporosis. Hormone Res 20: 74-81.

9 Naucler LO, Nilsson BE, Westlin NE (1974) An apparatus for gamma absorptiometry of bone. Technical data. Orpuscula Medico Technica Lundensia 12: (I) 1-14.

10 Finsen V, Haave $\varnothing$ (1987) Changes in bone-mass after tibial shaft fractures. Acta Orthop Scand 58: 369-71.

11 Whedon GD (1973) Comment in: Mazess RB, ed. International Conference on Bone Mineral Measurements. US Department of Health, Education and Welfare. NIH publication no. 75-683: 379.

12 Mirahmadi MK, Byrne C, Barton C, Penera N, Gordon S, Vaziri ND (1983) Prediction of creatinine clearance from serum creatinine in spinal injury patients. Paraplegia 21: 23-29.

13 Burr RG, Nuseibeh I (1985) Biochemical studies in paraplegic renal stone patients. Br J Urol 57: 269-74.

14 Perkash I, Martin DE, Warner H, Blank MS, Collins DC (1985) Reproductive biology of paraplegics: results of semen collection, testicular biopsy and serum hormone evaluation. J Urol 134: 284-88.

15 Naftchi NE (1985) Alteration in neuroendocrine function in spinal cord injury. Peptides 6 (Suppl 1): 85-94.

16 Claus-Walker J, Scurry M, Carter RE, Campos RJ (1977) Steady state hormonal secretion in traumatic quadriplegia. J Clin Endocrinol Metab 44: 530-535.

17 Phelps G, Brown M, Chen J, Dunn M, Lloyd E, Stefanick ML et al (1983) Sexual experience and plasma testosterone levels in male veterans after spinal cord injury. Arch Phys Med Rehabil 64: 47-52.

18 Pollock MA, Ratcliffe WA (1987) Laboratory comparison of three indices of serum free testosterone concentration. Ann Clin Biochem 24 (Suppl): 182-184. 